\title{
The development and genetics of digging behaviour in Drosophila larvae
}

\author{
Raúl Godoy-Herrera
}

Department of Cellular Biology and Genetics, Faculty of Medicine, University of Chile, Casilla 70061, Santiago 7, Chile.

Larval digging behaviour of Drosophila melanogaster and the sibling species Drosophila pavani and Drosophila gaucha differs quantitatively. Digging activity increases with age. Crosses between four strains of $D$. melanogaster indicate dominance and heterosis for digging; some non-allelic interaction is also suggested. Larval locomotion of a line with low digging behaviour is lower than that exhibited by larvae of a line with high digging behaviour. Larvae of the low digging line form puparia away from the medium, while those of the high digging line select places close to the substrate. However, larvae of a separate digger strain (the Oregon R-C strain) show similar locomotor behaviour to that of larvae of another non-digger strain (the vestigial strain). It is suggested that, besides locomotion, photoresponse of larvae may also influence the dispersal pattern within food. Dominance and heterosis towards high digging suggest possible evolutionary importance for this behaviour.

\section{INTRODUCTION}

Most animal species show a characteristic pattern of dispersal in the wild (Brown, 1975). Among Drosophila species, dispersal patterns of adult flies have been reported for factors such as food, reproduction and conspecifics (Spieth, 1974; Grossfield, 1978; Godoy-Herrera and Fenner, 1984). Few reports have been published on larval dispersal patterns in Drosophila, although burrowing is a behaviour common to a variety of Drosophila species. McCoy (1962) has found that with the exception of Drosophila busckii, larvae of Drosophila melanogaster, Drosophila hydei, Drosophila tripunctata and Drosophila quinaria, dig vigorously within overripe fruits of tomatoes.

MacArthur and Levins (1967) and Pianka (1982), have argued that natural selection favours organisms that specialise on a limited portion of the available resources which they exploit more efficiently than their competitors. In Drosophila, larvae of many species share similar nutritional requirements, one example being the sibling species $D$. melanogaster and Drosophila simulans (review in Ehrman and Parsons, 1981). Here I want to propose that larval digging behaviour of Drosophila is an adaptation to distribute themselves in space allowing optimal utilisation of resources such as food and pupariation places, preventing competition for these resources. Burrowing behaviour of $D$. melanogaster larvae seems to have a strong genetic component that persists under laboratory conditions (GodoyHerrera, 1977; 1978).

Larvae of Drosophila also show other behavioural responses, all of which may be connected with feeding and pupariation. Green et al. (1983) provide a description of the organisation of larval behavioural patterns in $D$. melanogaster and $D$. simulans. Larvae of $D$. melanogaster ingest food with a variable rate depending on age and genetic background (Sewell et al., 1975). Larvae of this species also show foraging responses (Sokolowski, 1980). All these behaviours are poligenically controlled (Burnet et al., 1977; Sokolowski, op. cit.; Sewell et al., op. cit, 1975). Besides, Godoy-Herrera et al. (1984) have found that young larvae of $D$. melanogaster show a preference for moist substrates, while mature larvae prefer a dry substrate on which to crawl. These authors have also shown that, in D. melanogaster, increase in larval locomotion with age is caused by growth in body size and changes in the genetic architecture of this behaviour. Sokolowski et al. (1984) have found that larvae of $D$. melanogaster of 3 to 4-days-old stay on food, but larvae at 
5-days-old tend to leave food for a more dry place; these behaviours are expressed sequentially during the larval development of this species.

The aims of this paper are to describe burrowing behaviour of larvae of $D$. melanogaster and the sibling species Drosophila pavani and Drosophila gaucha, and to provide a more detailed genetic analysis of digging behaviour by crossing four strains of $D$. melanogaster which show marked differences in digging (Godoy-Herrera, 1977).

\section{MATERIALS AND METHODS}

\section{(i) Stocks}

Drosophila pavani, Brncic, 1957. This species is endemic to Chile (Brncic, 1970). The strain used in this research was derived from wild flies collected in 1955 by Brncic (1957) at La Serena (Latitude $30^{\circ} \mathrm{S}$ ), and maintained since then by mass culture in the laboratory.

Drosophila gaucha, Jaeger and Salzano, 1953. The flies used were of the "Tainhas" strain kindly supplied by the Department of Genetics, University of Rio Grande do Sul (Brazil). The strain is also kept by mass culture in the laboratory.

These two species are sibling and make up the mesophragmatica group together with another six species all morphologically very similar (for details see Brncic, 1970).

Drosophila melanogaster, Meigen, 1830. The following strains were employed in this work: Oregon R-C (wild type), taxi $(t x)$, yellow $(y)$, and vestigial $(v g)$. Larvae of the high and low digging lines $\left(F_{20}\right.$ generation) derived from the Oregon $\mathrm{R}-\mathrm{C}$ strain by selective breeding (Godoy-Herrera, 1978) were also used (see below). All species and strains used here were kept in half-pint bottles filled with about $50 \mathrm{~cm}^{3}$ of Burdick's medium (1954) at $24^{\circ} \mathrm{C}$ with a constant regime of light.

\section{(ii) Vials with charcoal and egg collections}

Vials $2.5 \times 10 \mathrm{~cm}$ were filled with $4 \mathrm{ml}$ of culture medium mixed with finely powdered charcoal ( $2 \mathrm{gm}$ of charcoal $/ 100 \mathrm{ml}$ of medium). After coding, this medium was covered with $2 \mathrm{ml}$ of the same medium without charcoal. Depth of culture medium without charcoal was about $4 \mathrm{~mm}$.

The type of food used permits the recognition of two kinds of larvae hatched from the eggs laid over the food medium: (1) those that ingest medium with charcoal (named "Stained larvae" in the paper) and (2) those that eat medium without charcoal (denominated "Unstained larvae" in this work). Larvae which have eaten food with charcoal show a stained digestive tract under a stereomicroscope; those that have not fed on this medium exhibit a transparent digestive tract. Most of mature stained larvae ready to pupariate have particles of charcoal in their digestive tract, as seen in larvae crawling on the walls of the shell vials. Larvae showing a transparent digestive tract have presumably stayed eating in the upper zone of the food medium (for other details on the method see Godoy-Herrera, 1977; 1982).

Groups of $60-80$ inseminated females of the Oregon R-C, taxi, yellow and vestigial strains, and of the high and low digging lines were left without food for a 3 hour period in half-pint bottles. Starvation stimulates oviposition. After this period I introduced into each one of the bottles a plastic spoon filled with medium spread with fresh live yeast and acetic acid. These two substances stimulate oviposition. Eggs laid by females were collected after 3-4 hours with the aid of a dissecting needle. This was previously sterilised by flaming and cooling in alcohol to reduce the risk of contamination of the vials by fungi and/or bacteria.

\section{(iii) Digging behaviour}

One hundred and twenty vials with medium with charcoal (lower zone) and without charcoal (upper zone) were each sown with 30 eggs of the Oregon $\mathrm{R}-\mathrm{C}$ strain. Similar sets of these same vials were also each sown with 30 eggs of the taxi, yellow and vestigial strains. Eggs were carefully deposited over the surface of the nutritive medium to avoid scars with the needle. Young larvae seem to begin their burrowing activities through scars and/or holes in the surface of the culture medium.

The development of larval digging behaviour of the Oregon R-C, taxi, yellow and vestigial strains was measured as the percentage of stained larvae at $60,72,84,96,108$ and 120 hours of age. Very few larvae reach the lower zone before 60 hours. In each interval of time a set of 20 vials was examined. The same procedure was followed for studying digging behaviour of $F_{1}$ hybrid larvae produced by crossing the Oregon R-C, taxi, yellow and vestigial strains in all possible combinations. The arcsin transformation was used (Bartlett, 1947). Means of stained larvae of $F_{1}$ reciprocal crosses were statistically analysed for possible maternal effects or other kinds of reciprocal effects.

In the case of $D$. pavani and $D$. gaucha, mature 14-15-day-old females were used to collect eggs (Koref-Santibañez and del Solar, 1961). In these species the larval period at $24^{\circ} \mathrm{C}$ is longer than in 
D. melanogaster (Brncic et al., 1969). The number of stained and unstained larvae were recorded each 12 hours between 60 and 192 hours of development. In each interval of time 20 vials were examined. Each vial was sown with 30 eggs of $D$. pavani or $D$. gaucha. The development of digging behaviour in larvae of these two species was compared by taking the number of stained and unstained larvae at the different ages to calculate $\chi^{2}$ in $2 \times 2$ contingency tables (Sokal and Rohlf, 1969).

\section{(iv) Locomotor activity}

A rectangular $7.5 \times 14.5 \mathrm{~cm}$ piece of glass was covered with a layer of agar $3 \mathrm{~mm}$ thick. At 108 hours larvae of the Oregon R-C (digger strain), vestigial (non-digger strain) and the high and low digging lines were individually transferred onto the centre of the agar. Larvae used were reared in vials with medium without charcoal. Most of the larvae examined were picked off from the walls of the shell vials. After a settling-down period of 30 seconds larval locomotion was observed for 3 minutes and graphed using a camera lucida.
Length of the track made by each larva was measured by using a Hoffritz Map Measurer. Distance obtained for each track was converted into centimetres.

\section{(v) Pupariation of larvae of the high and low digging lines}

In each generation of selection $\left(F_{1}\right.$ to $\left.F_{20}\right)$ for high and low digging behaviour (Godoy-Herrera, 1978), two half-pint bottles, filled with $50 \mathrm{~cm}^{3}$ of medium without charcoal, were each sown with 200 eggs of each one of these lines. Distance between the surface of the medium and each one of 120 pupae were recorded at 148 hours of development.

\section{RESULTS}

\section{(i) Interspecific differences in digging}

Fig. 1 shows the development of digging behaviour in larvae of $D$. melanogaster, $D$. pavani and $D$. gaucha. At 60 hours of development about 8 per cent of larvae of $D$. melanogaster have dug down

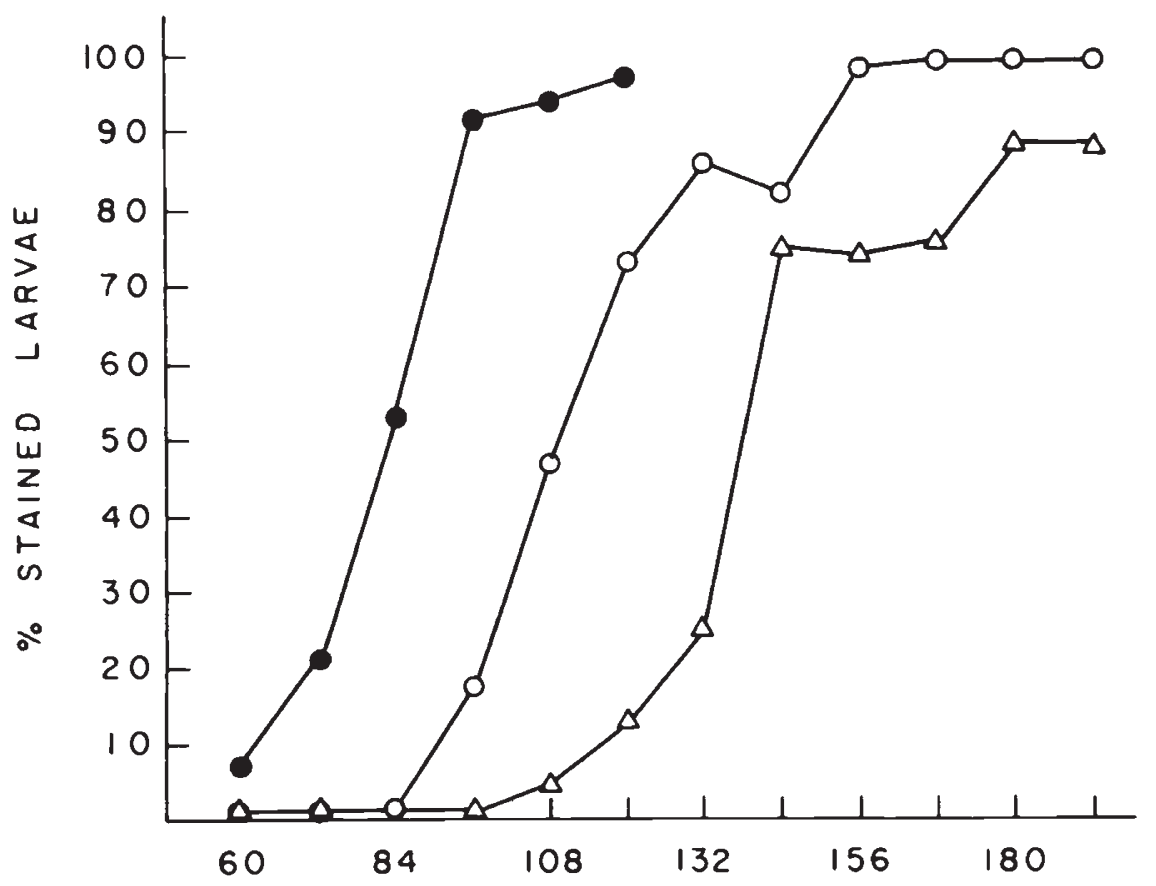

HOURS OF DEVELOPMENT

Figure 1 The development of digging behaviour in larvae of $D$. melanogaster $(O)$, D. pavani $(O)$, and $D$. gaucha $(\triangle)$ expressed as percentage of stained larvae at different larval ages (see Materials and Methods). 
to the medium with charcoal, while most of the larvae of $D$. pavani and D. gaucha stay in the upper zone of the medium. Between 72 and 96 hours the percentage of $D$. melanogaster larvae that have reached medium with charcoal progressively increases up to about 92 per cent; for 120 hours only 3 per cent of $D$. melanogaster larvae exhibit a clear digestive tract without charcoal.

Fig. 1 shows that at 96 hours of development 18 per cent of larvae of $D$. pavani have reached the medium with charcoal. After 144 hours of age 100 per cent of larvae of this species have eaten medium with charcoal. In contrast, the percentage of $D$. gaucha larvae stained with charcoal only increases after 108 hours of development; at the end of the larval period (192 hours of age) about 90 per cent of larvae of $D$. gaucha have burrowed down to medium with charcoal.

D. melanogaster has a larval period shorter than the sibling species $D$. pavani and $D$. gaucha. Comparisons between digging behaviours of larvae of these three species are difficult. In table 1 only digging activities of larvae of $D$. pavani and $D$. gaucha are statistically compared. It can be seen that with the exception of larvae at 144 hours of development, the pattern of larval dispersal of D. pavani within the medium is different from that of $D$. gaucha. These findings suggest that during the trophic stage of development $D$. pavani larvae consume food in zones of the substrate much deeper than $D$. gaucha larvae.

\section{(ii) Genetics of digging behaviour}

Fig. 2 (a, b, c) shows the development of digging behaviour in hybrid larvae obtained by crossing the two non-digger strains (the vestigial and yellow strains) with two digger strains (the Oregon R-C and taxi strains). Between 60 to 120 hours of development, $F_{1}$ hybrid larvae obtained by crossing the vestigial and Oregon $\mathrm{R}-\mathrm{C}$ strains dig within the medium like their digger parents of the Oregon R-C strain (Fig. 2 (a)).

Fig. 2(b) shows that $F_{1}$ hybrid larvae obtained by crossing vestigial flies (non-digger strain) with taxi flies (digger strain) also burrow into the culture medium as their digger parents of the taxi strain. On the other hand, $F_{1}$ hybrid larvae obtained by crossing two non-digger strains (the vestigial and yellow strains) tunnel into the substrate in a proportion greater than larvae of the yellow strain, indicating hybrid vigour for digging (fig. 2(c)).

Digging behaviour of $F_{1}$ hybrid larvae from crossing the yellow strain (non-digger strain) with the Oregon $\mathrm{R}-\mathrm{C}$ and taxi strains (digger strains) is shown in fig. 3 (a, b). After 72 hours of development $F_{1}$ hybrid larvae obtained from crossing the yellow and Oregon R-C strains dig medium like their digger parents of the Oregon $\mathrm{R}-\mathrm{C}$ strain (fig. 3 (a)). On the other hand, $F_{1}$ hybrid larvae from the cross between yellow and taxi flies show a burrowing behaviour greater than that of their digger parents of the taxi strain, suggesting again the existence of hybrid vigour for digging (fig. 3 (b); see also fig. 2 (c)).

Fig. 4 shows the development of digging behaviour in $F_{1}$ hybrid larvae originating from the cross between two digger strains: the Oregon $\mathrm{R}-\mathrm{C}$ and taxi. As seen in fig. 4, $F_{1}$ hybrid larvae dig into medium like larvae of the Oregon R-C strain. An inspection of means of stained larvae of each one of the $F_{1}$ reciprocal crosses studied in this

Table 1 The larval digging behaviour of the sibling species $D$. pavani and $D$. gaucha. Digging of the medium was measured for different time intervals. $\chi^{2}$ compares number of stained larvae of these species in each interval of time

\begin{tabular}{|c|c|c|c|c|c|c|c|c|c|c|c|c|}
\hline \multicolumn{13}{|c|}{ hours of development } \\
\hline & 60 & 72 & 84 & 96 & \multicolumn{8}{|c|}{ D. pavani } \\
\hline Unstained larvae & 184 & 247 & 385 & 355 & 152 & 79 & 71 & 50 & 9 & - & - & - \\
\hline Stained larvae & - & 3 & 6 & 95 & 146 & 264 & 355 & 290 & 411 & 396 & 330 & 359 \\
\hline$\%$ stained larvae & - & 0.01 & $1 \cdot 80$ & $18 \cdot 80$ & $48 \cdot 15$ & $74 \cdot 78$ & $87 \cdot 42$ & $86 \cdot 42$ & $97 \cdot 53$ & $100 \cdot 00$ & $100 \cdot 00$ & $100 \cdot 00$ \\
\hline Variance of stained larvae & - & $2 \cdot 39$ & $3 \cdot 95$ & $2 \cdot 22$ & 4.95 & $6 \cdot 82$ & $3 \cdot 22$ & $2 \cdot 01$ & $0 \cdot 28$ & - & - & - \\
\hline \multicolumn{13}{|l|}{ D. gaucha } \\
\hline Unstained larvae & 366 & 380 & 377 & 336 & 376 & 292 & 200 & 79 & 76 & 23 & 22 & 25 \\
\hline Stained larvae & - & - & - & - & 15 & 46 & 102 & 242 & 212 & 292 & 176 & 253 \\
\hline$\%$ stained larvae & - & - & - & - & $5 \cdot 52$ & $13 \cdot 20$ & 35.03 & $75 \cdot 37$ & $75 \cdot 12$ & $89 \cdot 83$ & $89 \cdot 11$ & $91 \cdot 00$ \\
\hline $\begin{array}{l}\text { Variance of } \\
\text { stained larvae }\end{array}$ & - & - & - & - & $1 \cdot 31$ & $4 \cdot 62$ & $7 \cdot 60$ & $3 \cdot 92$ & $9 \cdot 74$ & $1 \cdot 74$ & $1 \cdot 63$ & $1 \cdot 23$ \\
\hline \multirow[t]{2}{*}{$\chi^{2}$ test $(\mathrm{df}=1)$} & - & - & - & - & $19 \cdot 26$ & $27 \cdot 56$ & $18 \cdot 57$ & 1.03 & $9 \cdot 51$ & - & - & - \\
\hline & - & - & - & - & $\mathrm{p}<0.01$ & $p<0.01$ & $p<0.01$ & $p>0.05 p$ & $p<0.01$ & - & - & - \\
\hline
\end{tabular}




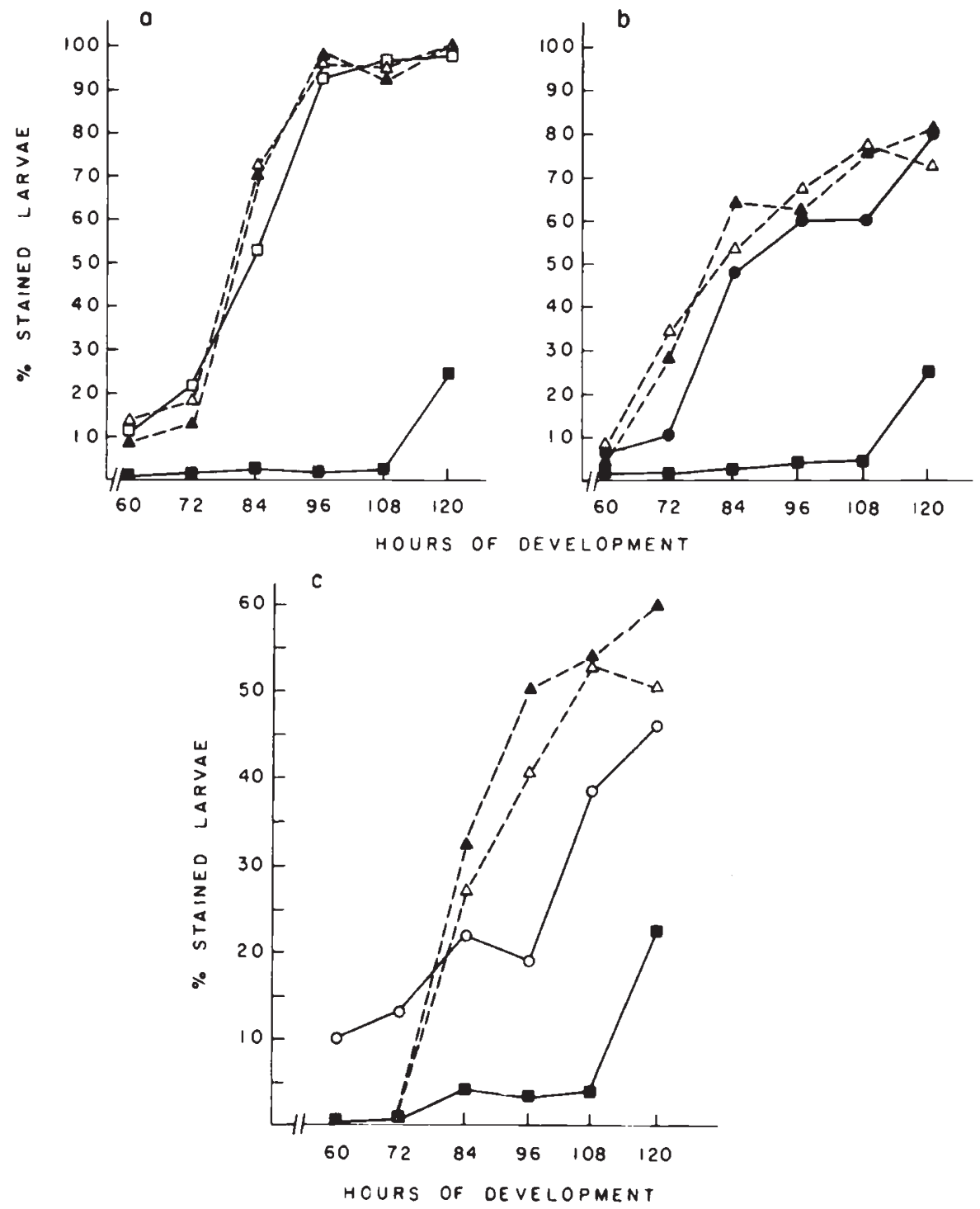

Figure 2 The development of digging behaviour in hybrid larvae from the cross of the vestigial ( $\square$ ) strain with the Oregon R-C $(\square)$, taxi $(\bigcirc)$ and yellow $(O)$ strains. (a) Oregon $\mathrm{R}-\mathrm{C}$ male $\times$ vestigial female $(\triangle)$; Oregon $\mathrm{R}-\mathrm{C}$ female $\times$ vestigial male $(\Delta)$. (b) taxi male $\times$ vestigial female $(\Delta)$; taxi female $\times$ vestigial male $(\Delta)$. (c) yellow male $\times$ vestigial female $(\Delta)$; yellow female $\times$ vestigial male $(\boldsymbol{\Delta})$.

research revealed no substantial differences between them (arcsine transformation). For instance, at 84 hours of development $t$ values ranged from 0.120 to $1.714(\mathrm{P}$, two tailed $>0.05)$; at 108 hours of development $t$ fluctuated between 0.029 and $0 \cdot 443$.

\section{(iii) Locomotor activity in larvae of digger and non-digger strains}

Burrowing behaviour entails considerable larval locomotor activity. Table 2 shows locomotor behaviour of larvae at 108 hours of development of the Oregon $\mathrm{R}-\mathrm{C}$ and vestigial strains and of larvae of the high and low digging lines (see Godoy-Herrera, 1978). Larvae of the Oregon R-C strain (digger strain) and vestigial larvae (nondigger strain) do not show substantial differences in locomotion. However, larvae of the low digging line show a locomotor behaviour lower than that of larvae of the base population (the Oregon R-C strain). Larvae of the high digging line exhibit higher locomotion than larvae of the unselected base population (the Oregon R-C strain). 

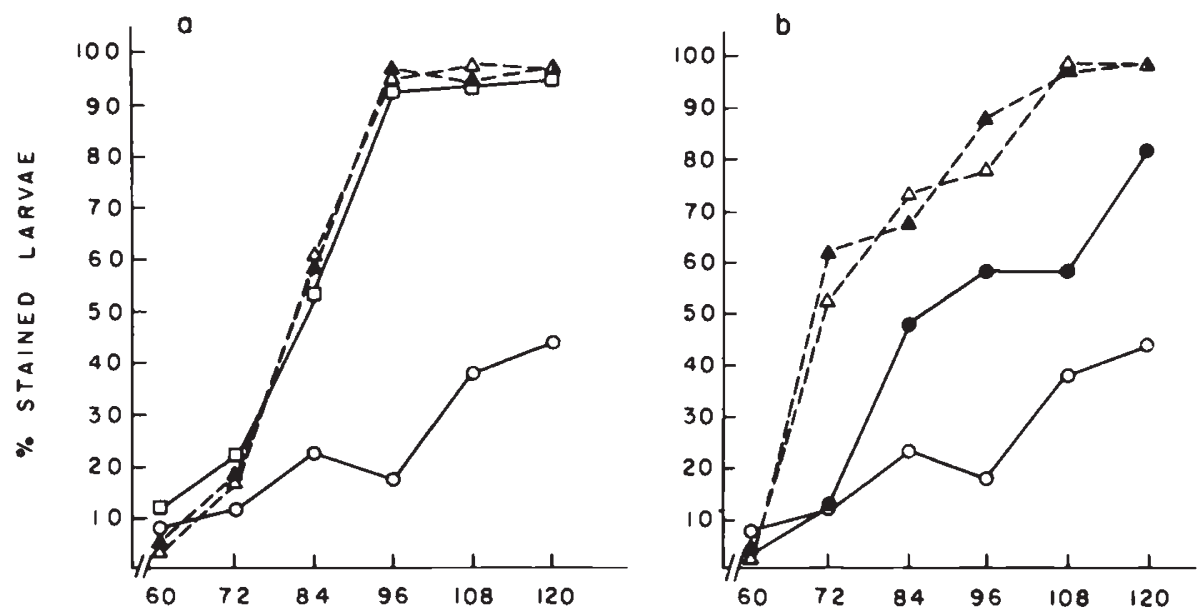

HOURS OF OEVELOPMENT

Figure 3 The development of digging behaviour in hybrid larvae obtained from the cross of the yellow (O) strain with the Oregon R-C $(\square)$ and taxi $(\mathbf{O})$ strains. (a) Oregon R-C male $\times$ yellow female $(\triangle)$; Oregon $\mathrm{R}-\mathrm{C}$ female $\times$ yellow male $(\mathbf{\Delta})$. (b) taxi male $\times$ yellow female $(\Delta)$; taxi female $\times$ yellow male $(\Delta)$.

(iv) Puparium forming behaviour of larvae of the high and low digging lines

Fig. 5 indicates that, after the F15 generation of selection for low digging, larvae of this line tend to form puparia away from the surface of medium, while those of the high digging line pupariate near

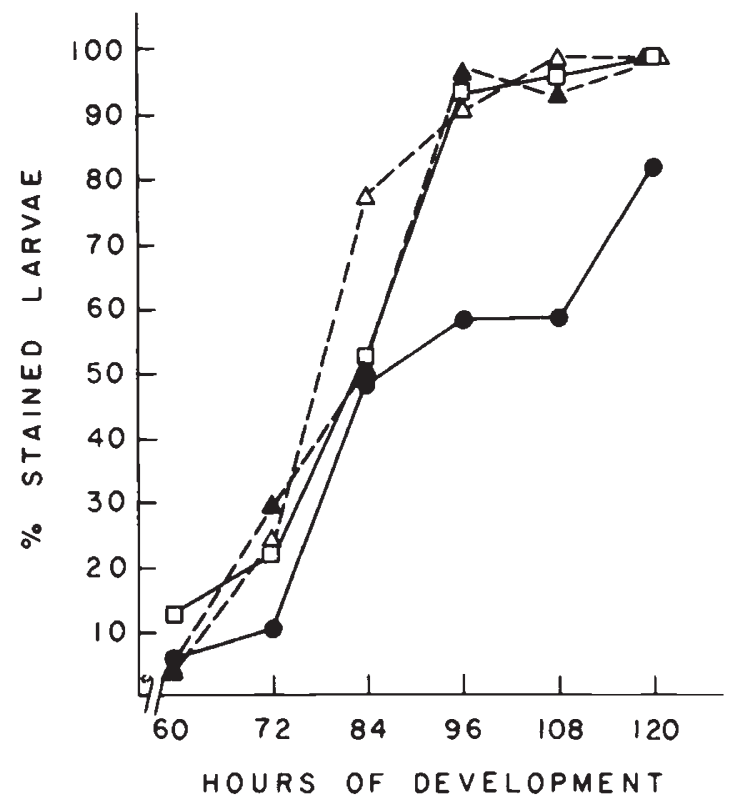

Figure 4 The development of digging behaviour in hybrid larvae obtained by crossing the Oregon R-C ( $\square$ ) and taxi (O) strains. Oregon R-C male $\times$ taxi female $(\triangle)$; Oregon $R-C$ female $\times$ taxi male $(\boldsymbol{\Delta})$. on the surface of medium, showing similar behaviour to that of larvae of the base population (the Oregon R-C strain).

\section{DISCUSSION}

This research shows that larval dispersal patterns of $D$. melanogaster within the substrate and of the sibling species $D$. pavani and $D$. gaucha depend on larval development (fig. 1 and table 1). In general, bigger older larvae seem to go into the culture medium deeper than smaller young larvae. In D. melanogaster, larval demands for food and

Table 2 Larval locomotor activity of the digger Oregon R-C strain and the non-digger vestigial strain and of the high and low digging lines (F20 generation). These last populations had been genetically selected from the Oregon R-C strain (see Materials and Methods).

\begin{tabular}{|c|c|c|c|c|}
\hline Strain & $\begin{array}{l}\text { Mean of } \\
\text { larval } \\
\text { locomotor } \\
\text { activity } \\
(\mathrm{cm})\end{array}$ & $\begin{array}{l}\text { Number } \\
\text { of } \\
\text { larvae } \\
\text { tested }\end{array}$ & $\begin{array}{l}\text { " } \mathrm{t} \text { " test } \\
\text { respect to } \\
\text { Oregon } \\
\mathrm{R}-\mathrm{C} \text { strain }\end{array}$ & $\begin{array}{l}\text { degrees } \\
\text { of } \\
\text { freedom }\end{array}$ \\
\hline Oregon $\mathrm{R}-\mathrm{C}$ & $47 \cdot 16 \pm 2 \cdot 46$ & 47 & - & - \\
\hline $\begin{array}{l}\text { high digging } \\
\text { line }\end{array}$ & $58 \cdot 20 \pm 2 \cdot 38$ & 50 & $3 \cdot 311^{*}$ & 95 \\
\hline $\begin{array}{l}\text { low digging } \\
\text { line }\end{array}$ & $38 \cdot 40 \pm 2 \cdot 06$ & 47 & $2 \cdot 811^{*}$ & 92 \\
\hline vestigial & $42 \cdot 00 \pm 2 \cdot 17$ & 31 & 0.466 & 76 \\
\hline
\end{tabular}

${ }^{*} p<0.05$ 


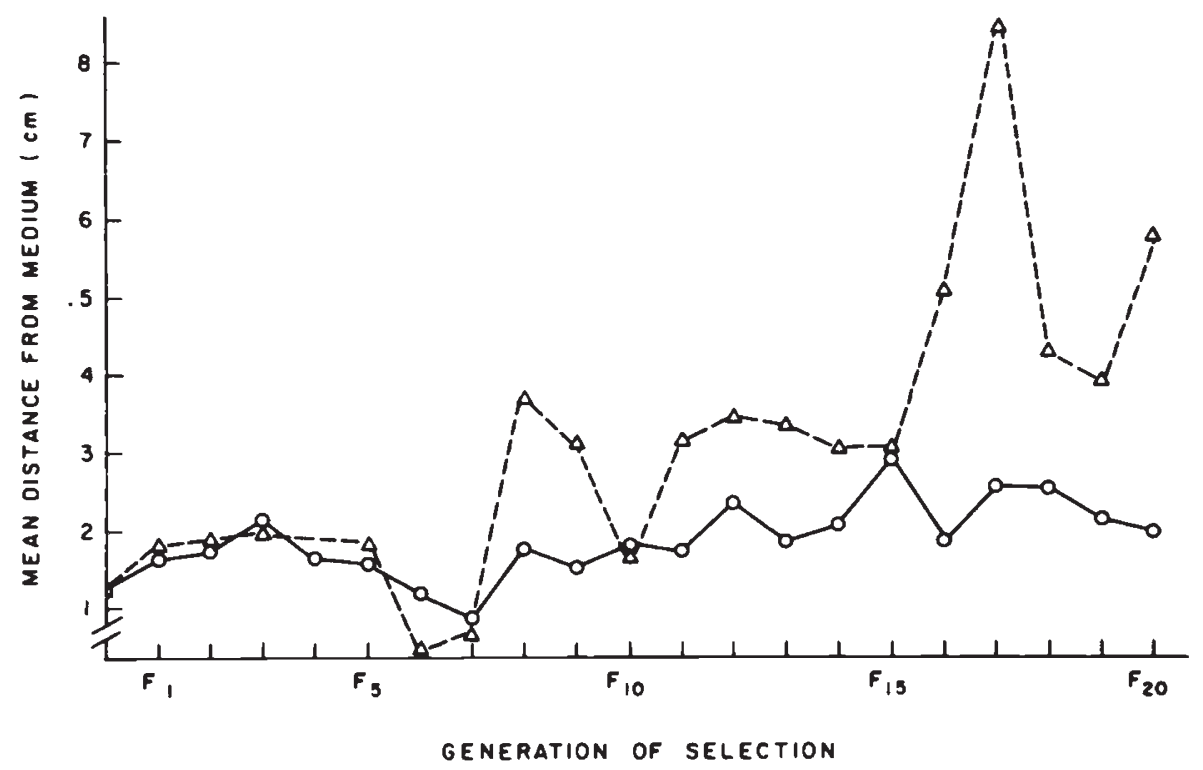

Figure 5 Changes in larval pupariation behaviour of the lines genetically selected for low $(\triangle)$ and high $(O)$ larval digging behaviour. Choice pupariation site was measured as a mean distance from the culture medium (see Materials and Methods).

space are also age-related (Sewell et al., 1975; Green et al., 1983; Godoy-Herrera et al., 1984; Sokolowski et al., 1984). Variations in digging shown by larvae of Drosophila with similar nutritional requirements could be of importance for understanding coexistence of these species in the wild (Barker, 1971). Atkinson (1979) has argued that competition for food and space between Drosophila species which breed at the same sites could be prevented through their larval behaviour which may lead to use of the resources of the environment in different ways.

When observing the number of larvae with and without charcoal, it was frequently noted that nearby or in the same tunnel made in the medium by a larva of $D$. melanogaster there could be found other individuals, suggesting that these preadults burrow in groups. Such larval aggregations were never observed in the case of $D$. pavani and $D$. gaucha. The viability of $D$. melanogaster larvae is not affected by their own biotic residues, but the residues of larvae of $D$. pavani and $D$. gaucha interfere with the development of these species (Budnik and Brncic, 1975; 1976). Individually digging the substrate might be a behavioural adaptation of larvae of $D$. pavani and $D$. gaucha which has evolved to decrease the effects of their own biotic wastes on viability.

Larvae of the Oregon R-C, taxi, yellow and vestigial strains and their hybrids also show digging behaviour related to age, as measured by the percentage of stained larvae between 60 to 120 hours of development. Digging of the culture medium is very fast between 72 to 96 -hours-old, decreasing after 108 hours (figs. 2 to 4). An increase of larval digging behaviour with age could allow for access to food at lower levels of the substrate expanding the space available to larvae, preventing larval inter- and intraspecific competition for these resources. The results also suggest dominance effects with some heterosis for digging (figs. 2(a), 2(b), 3(a) and 4), and that perhaps an epistatic component is present (figs. 2(c) and 3(b)). Characters closely related to fitness show a genetical architecture with allelic and non-allelic interactions and with little or no additive genetic variance (Broadhurst and Jinks, 1974). Godoy-Herrera et al. (1984) have shown that locomotor activity of $D$. melanogaster larvae increases with larval age as a result of growth in body size and of changes in the epigenetic relationships between genes controlling this behaviour.

Of the four strains of $D$. melanogaster studied in this paper Oregon R-C larvae showed a greater tendency to dig within the culture medium. This may reflect evolutionary pressures on digging behaviour of larvae of this strain. Such an inference is based on the fact that females of the Oregon $\mathrm{R}-\mathrm{C}$ strain are very prolific. Bottles in which the Oregon $\mathrm{R}-\mathrm{C}$ strain is cultured show numbers of larvae and pupae higher than those in which the taxi, yellow and vestigial strains are kept. These 
mutations decrease viability (Lindsley and Grell, 1972). The consistency of the culture medium is rapidly altered due to tunnelling by Oregon $\mathrm{R}-\mathrm{C}$ larvae, suggesting that a large number of these preadults are present. This suggests that larvae of the Oregon R-C strain may be under a continuous pressure to dig caused by high numbers of larvae hatching from eggs deposited by the females. Under continuous directional selection a gene would be expected to became dominant (Fisher, 1930).

Differences in locomotor behaviour may explain why there are differences in the amount of digging of the culture medium by larvae of the high and low digging lines (table 2). However, at 108 hours of age larvae of the digger Oregon R-C strain and those of the non-digger vestigial strain show a similar level of locomotion (table 2). I have found that larvae of the vestigial strain kept in darkness dig the culture medium substantially more than controls maintained in light (GodoyHerrera, 1982). Perhaps digging of the culture medium by $D$. melanogaster larvae may also be influenced by their photoresponse. More experiments are necessary to understand the relationships between larval age, locomotion and photoresponse in relation to the behavioural mechanisms of digging.

Selection for low digging behaviour has also modified pupariation behaviour of larvae of this line (fig. 5). Sokal (1966) reported that larvae selected to pupariate away from medium tend to remain in the upper zone of substrate when feeding. These findings suggest that genes controlling these two larval behaviours of $D$. melanogaster could be linked on the same chromosomes. On the other hand, the fact that larvae with a comparatively low locomotor activity (table 2) pupariate away from the medium confirms that other factors such as humidity are important in selection of pupariation sites (Sameoto and Miller, 1968; Schlager, 1960).

Acknowledgements I am indebted to Dr Barrie Burnet for commenting on and correcting the manuscript and Dr Danko Brncic for flies and discussions. I also thank Lilya Milla for her secretarial help. This research was supported by Grant 1619-8423 from the Universidad de Chile.

\section{REFERENCES}

ATKINSON, W. D. 1979. A comparison of the reproductive strategies of domestic species of Drosophila J. Anim. Ecol., $48,53-64$.
BARKER, J. S. F. 1971. Ecological difference and competitive interaction between Drosophila melanogaster and Drosophila simulans in small laboratory populations. Oecologia, 8, 139-156.

BARTLETT, M. S. 1947. The use of transformations. Biometrics, 3, 39-52.

BRNCIC, D. 1957. Las especies chilenas de Drosophilidae. Imprenta Stanley, Santiago de Chile.

BRNCIC, D. 1970. Studies on the evolutionary biology of chilean species of Drosophila. In M. K. Hecht and W. C. Steere (eds), Essays in Evolution and Genetics in Honor of Th. Dobzhansky. Appleton-Century Crofts, New York.

BRNCIC, D., KOREF-SANTIBAÑEZ, S., BUDNIK, M. AND LAMBOROT, M. 1969. Rate of development and inversion polymorphism in Drosophila pavani. Genetics, 61, 471-477.

BROADHURST, P. L. AND JINKS, J. L. 1974. What genetical architecture can tell us about the natural selection of behavioural traits? In J. H. F. van Abeleen (ed), The Genetics of Behaviour. North-Holland Pub. Co., Amsterdam.

BROWN, J. L. 1975. The Evolution of Behavior. Norton, New York.

BUDNIK, M. AND BRNCIC, D. 1975. Response of Drosophila pavani, Drosophila gaucha and their hybrids to larval biotic residues. Experientia, 31, 781-782.

BUDNIK, M. AND BRNCIC, D. 1974. Effects of larval biotic residues on viability in four species of Drosophila. Evol ution, 29, 777-780.

BURDICK, A. B. 1954. New medium of reproductive quality stable at room temperature. Drosoph. Inf. Serv., 28, 170.

BURNET, B., SEWELL, D. AND BOS, M. 1977. Genetic analysis of larval feeding behaviour in Drosophila melanogaster. II Growth relations and competition between selected lines. Genet. Res., 30, 149-161.

EHRMAN, L. AND PARSONS, P. A. 1981. Behaviour Genetics and Evolution. McGraw-Hill, New York.

FISHER, R. A. 1930. The genetical Theory of Natural Selection. Oxford, Clarendon Press.

GODOY-HERRERA, R. 1977. Inter- and intrapopulational variation in digging in Drosophila melanogaster larvae. Behav. Genet., 7, 433-439.

GODOY-HERRERA, R. 1978. Selection for digging behavior in Drosophila melanogaster larvae. Behav. Genet., 8, 475-479.

GODOY-HERRERA, R. 1982. Genetic-Ethological and evolutionary studies on digging behaviour of Drosophila (Diptera, Drosophilidae). Ph.D. Thesis, Faculty of Basic Sciences and Farmaceutics of the University of Chile (in Spanish).

GODOY-HERRERA, R., BURNET, B., CONNOLLY, K. AND GOGARTY, J. 1984. The development of locomotor activity in Drosophila melanogaster larvae. Heredity, 52, 63-75.

GODOY-HERRERA, R. AND FENNER, H. 1984. Behavioral responses of Drosophila pavani adults to other members of the population. Brazil. J. Genetics, 7, 41-54.

GREEN, C. H., BURNET, B. AND CONNOLLY, K. 1983. Organization and patterns of inter- and intraspecific variation in the behaviour of Drosophila larvae. Anim. Behav., 31, 282291.

GROSSFIELD, J. 1978. Non-sexual behavior of Drosophila. In Ashburner, M. and Wright, T. R. F. (eds). The Genetics and Biology of Drosophila, Vol 2b, Academic Press, London.

KOREF-SANTIBAÑEZ, S. AND DEL SOLAR, E. 1961. Courtship and sexual isolation in Drosophila pavani Brncic and Drosophila gaucha Jaeger and Salzano. Evolution, 15, 401406. 
LINDSLEY, D. L. AND GRELL, E. H. 1972. Genetic variations of Drosophila melanogaster. Carnegie Inst. Wash. Publ., 627.

MCARTHUR, R. H. AND LEVINS, R. 1967. The limiting similarity, convergence, and divergence of coexisting species. Am. Nat., 101, 377-385.

MCCOY, C. E. 1962. Population ecology of the common species of Drosophila in Indiana. J. Econ. Ent., 55, 978-985.

PIANKA, E. 1982. Ecología evolutiva. Omega, Barcelona.

SAMEOTO, D. AND MILlER, R. S. 1968. Selection of pupation site by Drosophila melanogaster and Drosophila simulans. Ecology, 49, 177-180.

SCHLAGER, G. 1960. Controlling fluctuations of pupation site in Drosophila. Trans. Kansas Acad. Sci., 63, 243-257.
SEWELL, D., BURNET, B. AND CONNOLLY, K. J. 1975. Genetic analysis of larval feeding behaviour in Drosophila melanogaster. Genet. Res., 24, 163-173.

SOKAL, R. R. 1966. Pupation site differences in Drosophila melanogaster. Univ. Kansas Science Bull, 46, 697-715.

SOKAL, R. R. AND ROHLF, F. J. 1969. Biometry. Freeman and Company, New York.

SOKOLOWSKI, M. B. 1980. Foraging strategies of Drosophila melanogaster. A chromosomal analysis. Behav. Genet., 10, 291-302.

SOKOLOWSKI, M. B., KENT, C. AND WONG, J. 1984. Drosophila larval foraging behaviour: Developmental stages. Anim. Behav., 32, 645-651.

SPIETH, H. J. 1974. Courtship behaviour in Drosophila. Ann. Rev. Entomol., 19, 385-405. 